\title{
D. D.
}
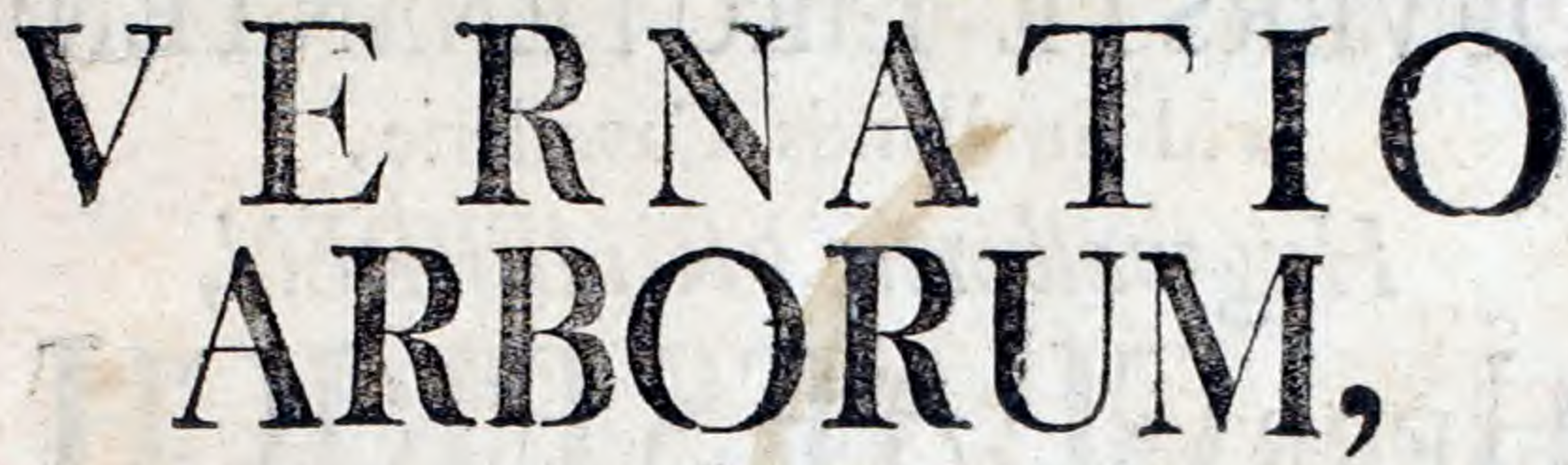

QUAM,

CONSENT. AMPLISS. FACULT. MEDICA;

IN REGIA ACADEMIA UPSALIENSI,

\section{Sub PR FSIDIO}

VIRI NOBILISSIMI,

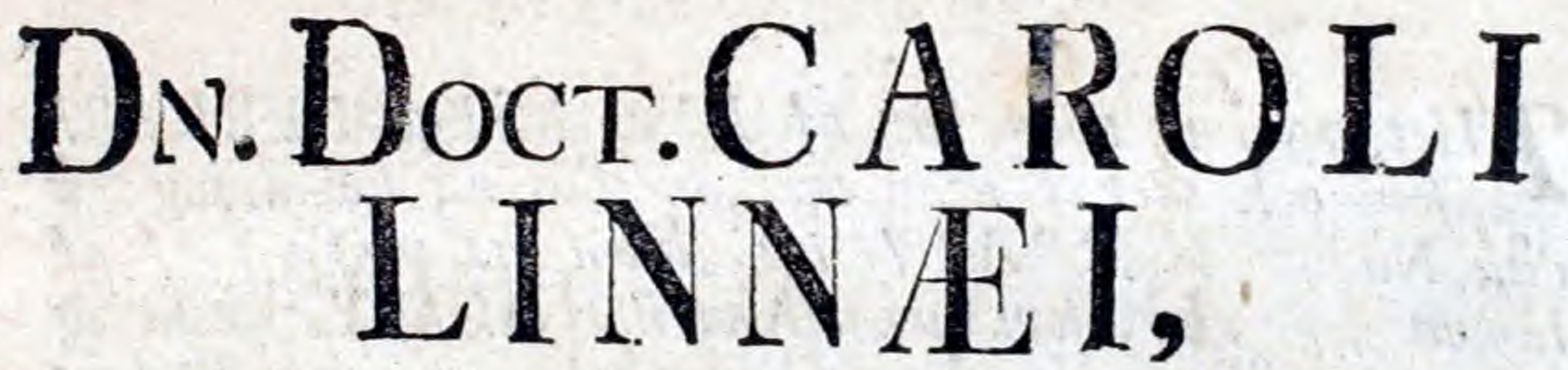

S:IE R:E M:TIS ARCHIATRI,

Med. et Botan. PROFESS. Reg. et ORd.

Acad. Imper. Monspel. Stockholm.

Upsal. Berol. et Tol, SOCII,

PUBLICO EXAMINI SUBMITTIT,

HARALDUS BARCK,

SMOLANDUS.

IN AUDIT. CAROL: MAJ. D. V. MAJI,

ANN. MDCCLIII.

H. A. M. S.

UPS A L I $\mathbb{E}$,

Exc. L. M. HO̊JER, Reg. Acad. Typogr. 


\section{Kongl. Maj:ts TRO- O̊FVERSTE-LIEUTENANTEN wid Smâlands Cavallerie, Hogwalborne FRIHERREN,

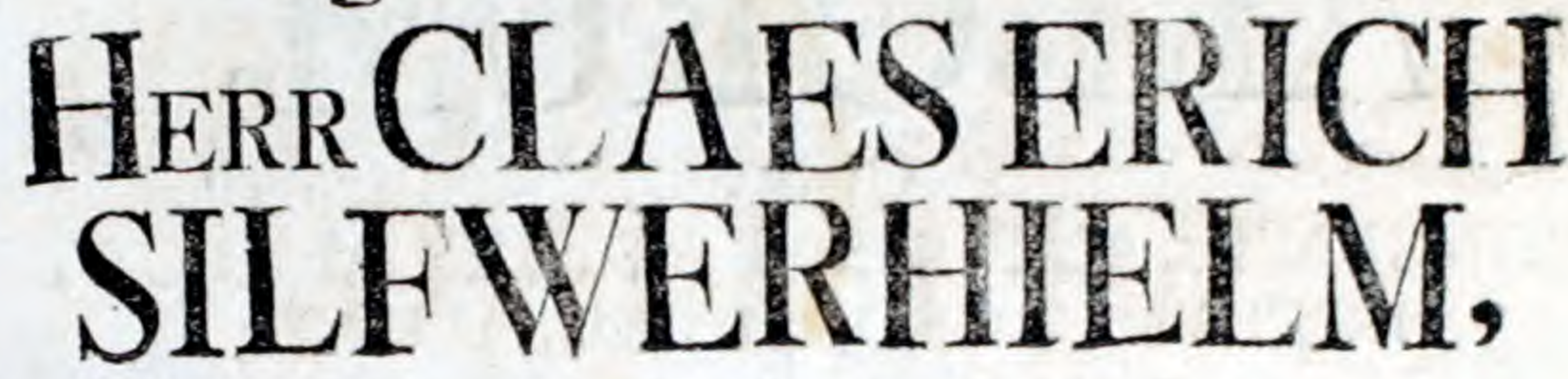

\section{Nádige}

THet myckna gode, jäg of Eder, Nadiga HERRAR! 1 fom ock therat utan twekan fluter, bwad jig bi ia, Nådige HERRAR! att la Jom ett ringe prof af lámna defe ron, lom jag til en Aor del Famlat omkring ocb underballit mitt neje: Eder forfarenbet bar Atadgat dà de antagit den grona ork tả ka wairdránaten.

Tilliot $n$, Nadige HERRAR! mo, dà jng efter tberinunder af trâns loffning ut for ka reitta Säningstiden, Wägren mig ej, att pá thetta enda láttet, jag jag, in till mitt friffa bar den ciran att frambar

\section{Mine Nâdige \\ $\mathrm{ED}$}

Edmirkafte HARAL: 
I J EN A R E,

MAJOREN Och RIDDAREN af Swärds - Orden,

Wiblborne HERREN,

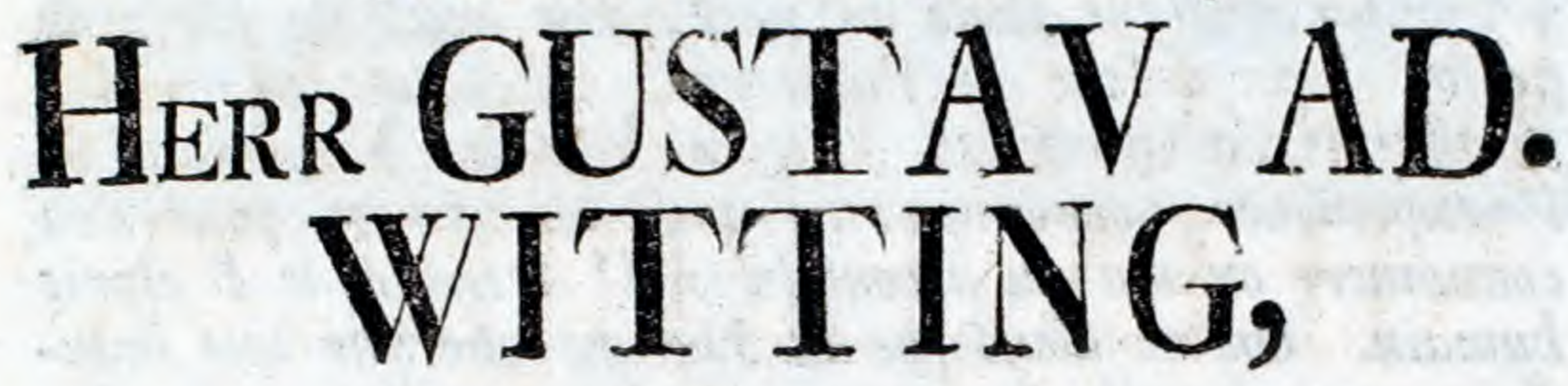

\section{HERRAR!}

redan ätnjutit, wordar jag med odmiukefta tack/nmbet: danefter of Eder ynneft bar att wánta. Tillädjen mig en undcrgifwen wordinad framte och uti Edra binder Edra Sátesgårdar. Eder upmairk/ambet bar wäckt min nyfikenbet, til att åkeda trän ocb antekna tiden,

frátwar ranlake naturen och télia des Jporr, laint att Jokk ocb finna kygd och ; for war bos Eder.

tormar, betyga den ofkryintade wordnad, bwarmed da,

\section{HERRAR,}

ER

tjerare, BARCK. 


\section{MONSIEUR,}

Or conviendra lans difficulté quc les fciences font reduites à ce degré de perfection, que celui qui veut y ajouter quelque chole de réel, doit avoir la force de penfer, au deffus du Vulgaire. Elles font neanmoins Jufceptibles de progrés, c'eft ce que les Exemples E? $l$ 'experience nous montrent tous les jours: pour s'en convaincre on n'a qu'à confiderer l' etendue de l'elprit bumain. On ne developpe la Nature que par une induArie, qui meprile les travaux \& tes veilles. Le Public, MONSIEUR, Vous jern indubitablement obligé de l' ouvrage que Vous lui allés communiquer. Vous avés choif \& expliqué une matiere, qui porte temoignage de Vos talens o de Vos lumieres. Fe Vous fuis trop redevable, MONSIEUR, pour la1ger ecbapper une occafion fi favorable de Vous en teliciter du meilleur de mon coeur. L' appiaudifement du Public à l' egard de cet effai ne lera qu' wn interprête des recompenjes que Vôtre vertu merite.

F'ai l' bonneur d'être,

\section{MONSIEUR,}

Votre trés affectionné Er trés fidéle lerviteur, ADAM GUST AV WITTING. 


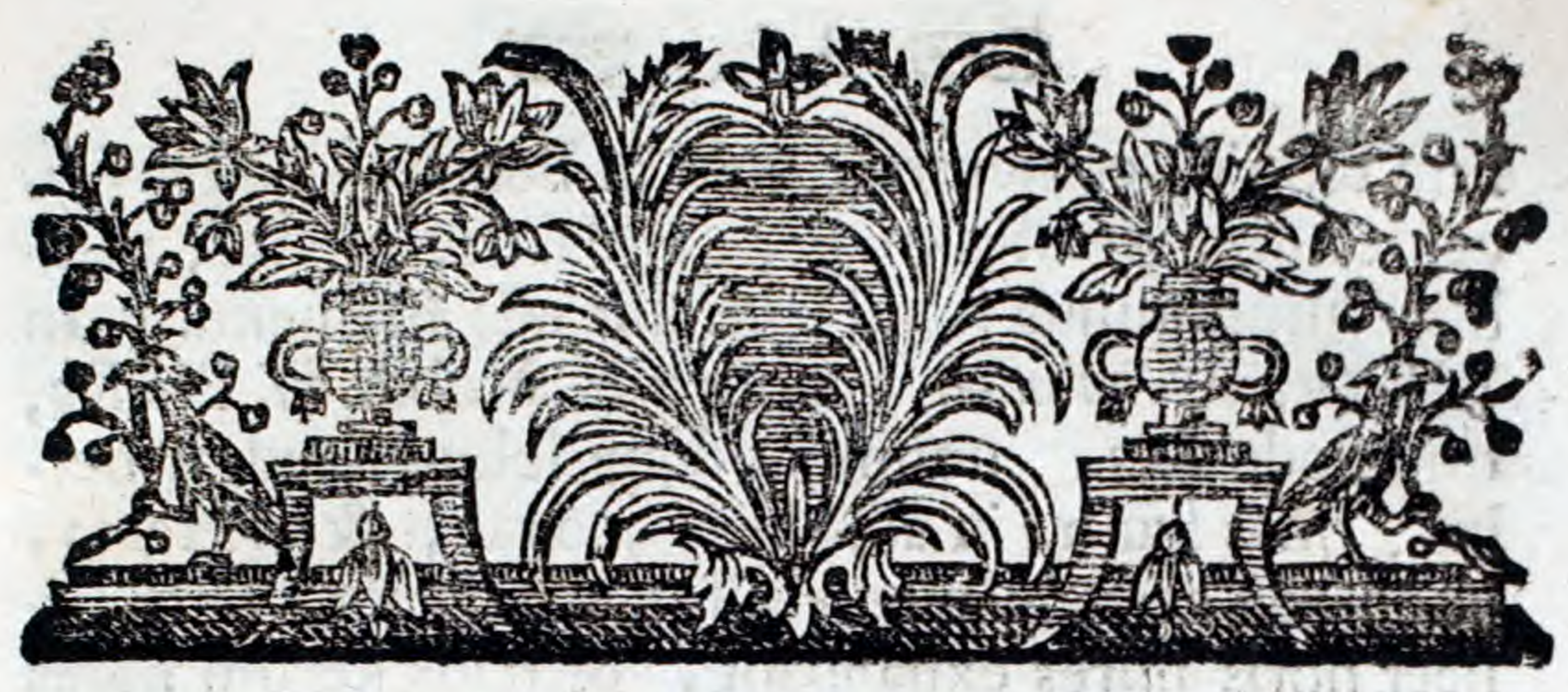

\section{Q. D. B. V. \\ 6. I.}

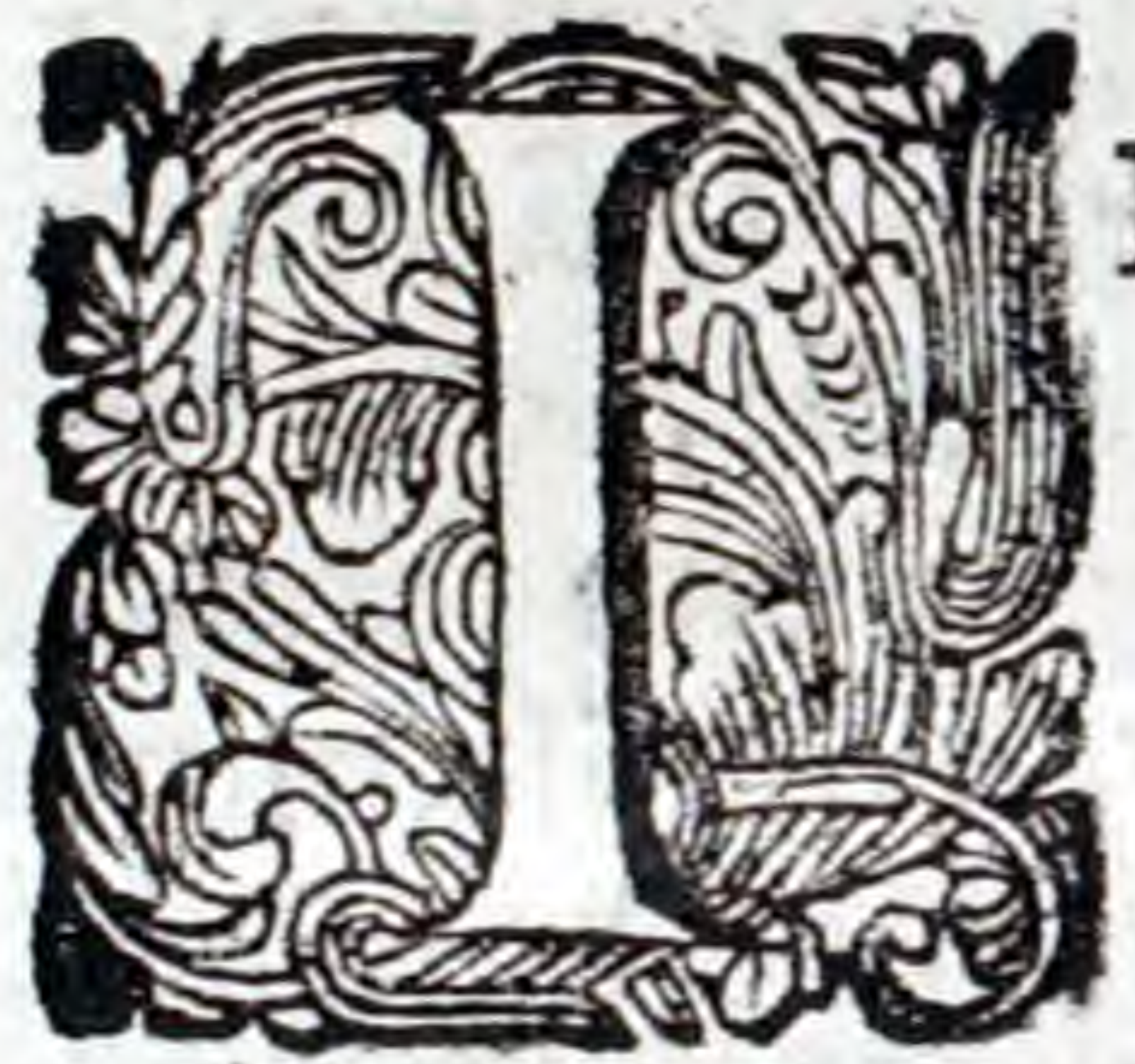

$\mathrm{N}$ eo defudarunt omnis ævi Botanici, ut plantas non detegerent folum \& nominarent ; fed, qua fieri potuit, etiam defcriberent diligentia. Verum enim vero jufto, quod mireris, arctioribus terminis circumfcripta erat in præfens usque feculum hæc fcientia; ea enim opinio erudicorum fere omnium animos inceflerat, non extra medicinæ campum eius patere ufum; unde tune ejus cultores præter Efculapii filios, paucos admodum videbis, nec ultra mediocre herbarum officina. lium ftudium eorum progreffam deprehendes indufriam. Noftris autem temporibus, vires plantarum majori cura \& induftria exquirere \& perveltigare conati fane Viri, in orbe botanico nunquam fatis collaudandi. Hi enim, præter medicum ufum, alias hujus ftudii magnas \& præclaras detexerunt utilitates, quibus percenfendis jam fuperfedeo, verbo fo. 


\section{(6er3) $6($ s)}

Jum tetigiffe contentus, quid in Botanico noftri Lycei campo preftitum fit, unde fatis, opinor, pate. bit, ufum hujus ftudii non vilis effe momenti. In Pbilofopbia Botanica monftravit Nobil. D. Præfes, quomodo fuis, unumquodque folum gaudeat herbis, quas fruftra in aliis locis quæras, \& præterea, quomodo certæ plantæ vigilias fuas agant, ftatis fcilicet horis flores expandant, \& Itatis iterum flores fuos occludant (a). Differtatio de Sponjaliis planta. rum, ufum variorum, quæ in foecundatione obfer. vare licet, phœnomenorum, cum orbe erudito communicavit. Flora Oeconomica, ufum plantarum in ceconomia privata fideliter aperuit. Diflertatio de Gensmis Arborum nobis cauflam patefecit, cur variæ arbores, noftri orbis nives \& frigora non ferant. Ex Difputatione de Plantis E/culent is Patrice difcimus, varias in noftro orbe crefcere plantas, quæ humano corpori alimenta præbere poffint. In Pane Suecico. videmus, certas tantum herbas certis animalibus efui efle deftinatas. Ex Holpita Infectorum Flora colligimus, quomodo certa vegetabilia certas infectorum fpecies alant \& nutriant.

Quartus jam agitur annus, ex quo Populares noftros in novellis literariis exhortatus eft Nob. D. Præfes, ut omni cura \& diligentia obfervarent, quo qualibet arbor tempore fuas expandat gemmas, fo. liaque explicet, non vana innixus divinatione, futurum, ut ex pluribus hujusmodi, variis in locis, inftitutis obfervationibus, novum \& forte non exfpe.

Etatum

(a) Vid.Pbil. Bot.pag. 263; 273. 


\section{(घ) ) ๆ}

Etatum fruetum noftra capiat patria. Quo monitu ego, qui eo tempore in Smolandia commorabar, apud Nob. Dom. G. A. WitTing, Majorem \& Equitem de Oddine militari, incitatus, per fpatium trium annorum, nempe ab initio anni MDCCL. ad idem tempus currentis, Dies, quo arbores frondes emittere ccepiffent, annotavi; nec non quo tempore agros confeverint ruricolx, \& quantum denique temporis fpatium fementem inter \& meffem interfuerit. Hxc eapropter a me facta effe fcias, ut certas inde definire poffem leges, quibus in qualibet provincia fementis effet attemperanda. Sed non paucx, quas mihi inftituere licuit, obfervationes huic fuffecerint negotio; itaque ne incerto \& lubrico fundamento res, quam meditor, fulciretur, non gravatus eft Nob. D. Prafes omnes fched ulas, ex diverfis ad Se transmiflas locis, mihi concedere excutiendas. In alio, fateor equidem, argumento, mea defudavit induftria; fcilicet animus fuerat Floram Gripenbergenfem, omnium crrca mite Gripenbergæ folum crefcentium plantarum ufum, cum medicum, tum ceconomicum complexuram, loco Speciminis Academici, publici juris facere; fed cum perpaucis ifthanc operam ufui futuram fuifle deprehenderem, mutato confilio, prafentem elegi materiam, utpote quam Parrix non ingratam fore judi. caverim. Tuum ergo erie, B. L. hofe conatus miciori cenfuræ fubjicere.

\section{II}

Terra noftra, quam frigidum ccelum urget, hiber- 


\section{(e) $) 8($}

hiberno tempore gelı concrefcit, hinc radices pigro quafi fopore preffæ torpent, plurimæque herbæ, fupra terram exftantes, emoriuntur $(b)$. Cum autem fol, mizibus fuis radiis, fub veris initia, eam re. creat; diffugiunt nives, glacies liquefcit, gelu folvitur \& lata redit rerræ facies. Vernos flores ftatim videmus fuas celebrare nuptias, arboresque, alte. ram polt alteram, gemmas referare foliisque amiciri. Mirari certe convenit cauflam, cur plantæ NEMOROSE, uti Daphne, Anemone Nemorofa, Hepatica, Orobus Vernus, Latbraa, Pulfatilla, Tufilago, Ranunculus Ficaria, Viole \&c. \& HOR TORUM, Galan. thus, Leucojum, Crocus Vernus \&c. primo vere florefcant, quas nulla opera nullaque cura eo redigere poflumus, ut fub autumnum feu polt folftitium æefti. vum, in florem fe induant. Notatu etjam dignum eft, has herbas, verno licet tempore, frigoris patientiffimas; autumno tamen adeo molles effe \& fragiles, ut, more Indicarum herbarum, ad primam pruinam emoriantur (c). e. gr. Soncbus Alpinus, im.

(b) Ouinque pralertim in Svecia numerantur HIEMES

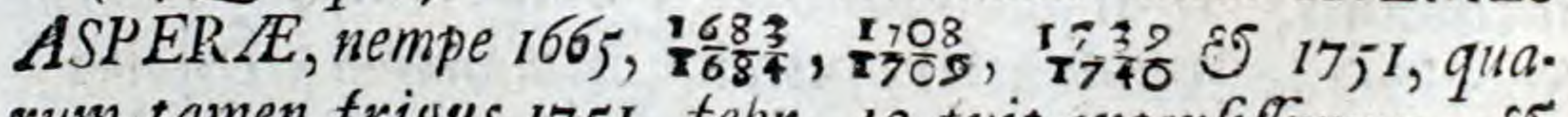
rum tamen frigus 1751 febr. 12 fuit intenfifimum, of vix boc feculo apud nos ob/ervatum, dum frigus ad gr.32 in thermometro Botanico defcendebat.

(c) NOCIES FERREAE vulgo dictæ Sv. Färnnätter, ut plurimum incidunt heic Upfaliz inter VIII \& XX mienfis Augufti, e.gr. $17468,17486,174921,175019$. 175116,17529 , \& ultra duas aut tres noctes 


\section{(15) $) 9(-5$}

patiens \&c. CAKDUOS non ante folltitium efflorefces re cernimus, unde ruricolæ ex eorum flore, tanquam ex calendario, fallere nefcio, judicant folftitium præteriifle. Hinc patefcit, aliud quidem efle, præter aquam \& calorem, quod plantarum promo. veat fertilitatem.

\section{S. III.}

Pari ratione fixas leges certumque ordinem in foliatione obfervant arbores, unde fit, ut quilibet, in hac re vel mediocriter verfatus, dum unam videt arboris fpeciem frondefcere, ftatim fciat, quænam proxime poft eam fuas explicare debeat frondes. Nec facile ullam obfervabimus arborem hunc FLO. B

RIE

raro durant. Poft hafce non amplius crefcit Hordeum, nec hifce inftantibus fuas Hefperides aliasque molliores herbas libero diutius aèri credere audent hortulani. Tunc uruntur folia Ficls, Mori, Iuplanilis, Vitis, Toxico. dendri, ipfiusque Fagi; INDIC E plantæ Pbalenti, Ta. getes, Cucumeres, Amaranti, Convolouli, Nicotiane, Dature, Ambrofie, Fagopyra, Borragines, Perficaria oricntalis, Milum, Tropealum, Balfamina, Lycoper. ficum, Pbyfalides amnue, Sicvos, Mirabrlis, Heliantbus annutus, Oenatbera, Aralia, Eupatoria virginia. na, Heliotropium, Botrys, Portulaca, Laduca \&c. emoriuntur, immo ipfa interdum INDIGENAE Impatiens, Xantium, Bryonia, Echium, Anagallis, Soncbus alpinus, Chenopodium rubrum \&c. marcetcunt. Prius vero quam hæc fiunt, explicat Colchicum fuos flores, idque interdum ferius, interdum prius, prout vel tardiores vel citiores fuerint ferreæ hæ noctes. 


\section{(49) ) 10 ( )}

RE ORDINEM transgredi. Hujus autem rei veram cauffam fruftra in eo quæfiveris, quod una arbor alcioribus altera nitatur radicibus ; tunc enim prius frutices, quam arbores unius ejusdemque fpeciei, frondes femper emitterent, qued tamen raro fit, fed fluit fine dubio hoc, ex alia quadam cauffa, nobis adhuc incognita, \& ex interna forIan arboris textura derivanda. Ordo, quem fervant arbores, folia explicaturæ, hic fere eft, nempe:

I Groffular. A. So. 195.

2 Ribes - $19 \%$.

3 Padus - 396.

4 Sambucus - 250.

5 Sorbus -400.

6 Salix $-\quad 812$

7 Alnus $=775$.

8 Malus - 402 .

9 Cerafus Hort. -

10 Opulus fl. Siv. 249.

11 Bitula - -776.

12 Corylus - -877 .
13 Ulmus f. Sv.

14 Rora - $=496$

${ }_{15}$ Pyrus - 401

16 Prunus bort.

17 Rhamn. A. Sv. 195

18 Frangula - 194

19 Tilia - -432

20 Fagus -785

21 Cratægus - 398

22 Populus - 819

23 Acer - - 303

24 Quercus - 784

25 Fraxinus - 830

Primarum jam enumeratarum arborum $(1,2,3,4)$ frondefcentia, qua tempus \& diem, quo folia e. mittunt, multum variat, prout enim hiems citius vel ferius mitefcit; ita hæ temporius vel tardius frondefont; reliquarum vero non item. Ex. gr. Anno MDCCL quo hiemis vis in Svecia auftraliore prorfus nulla fuit, fed continuum tere ver, obfervavi ultimis diebus nenfis Februarii Ribem \& Groflulariam circa Gripenbergam forefeere, 


\section{(5) IIC cos}

praterito autem anno demum initio menfis Apric lis; Quercus vero una cum Frasino, folia fua raro nobis prius oftendunt, quam gelu nocturnum fe. re cellaverit (d). Unde hortulani hefperides fuas; cœlo prius committere non audent, quam folia ultimarum arborum figna eis præbuerint mitioris; tempertatis.

\section{f. IV.}

Opportunum, fi quod aliud, Seminandi tempus, maxima cura obfervabit prudens ceconomus; id enim, fecundum benedictionem divinam, ubertatem facit annonæ, \& fundamenta ponit, cum publicæ totius regni falutis, tum privatæ civium felicitatis. Ruricola, patriorum ricuum \& inveteratæ confvetudinis jufto tenacior, fementem ut plurimum certo menfi, tempori \& diei alligat; utrum vero nec ne terra ad femen excipiendum parata fit, parum penfi habet, unde non raro accidit, ut arva fallant depofitum, \& quod cum fudore feminat feminator, cum mcerore demetere coga. tur meflor. In eo ergo maxime laborarunt o. mnis ævi Oeconomi, ut certum definirent \& fixum fationis tempus, fed hactenus irritus fuit 0 mnis eorum labor. Fuere, qui ex gufu vel ol.

$$
\mathrm{B}_{2}
$$

factu

(d) Ultra decennium obfervatum eft, QUERC UM fua Vernatione merfern $M a j i$ non transgrefiam fuife in $\mathrm{U}_{\mathrm{P}}$ landia; patet ex annis 1741 22,1744 8,1747 9,175016,

$$
\begin{aligned}
& 1742 \text { 3, } 174512,174813,175118, \\
& 17436,174610,1749-175^{2} 18
\end{aligned}
$$




\section{(5) ) $12(\cos :$}

factu terræ qualitates, ad hunc finem obtinendum neceflarias, perveftigare conati fint; nec illi defuere, qui fibi perfvaferint, feerorem terræ vel fla Dive Virginis, certiffima fore fementis indi. cia. Quæ licet omnia fuo forte non careant fundamento, fini tamen, quem quærimus, obtinen. do nequaquam fufficient. Multorum enim anno. rum experientia docuit, femina unius ejusdemque fpeciei, eidem quidem agro, fed diverfo tempore, commifla, non xque uberes tulife fruetus. Sic etiam dum una planca viget \& florefcir, altera ejusdem indolis \& in eudem nata folo marcefcit \& emoricur. Cauflam fterilitatis haud raro con. jiciunt agricolæ in Sanctiffimum Numen, ingratam puniturum gentem, agros interdum jubens 1pontaneis herbis horrefcere, fegetesque ludere co. lonos inanibus ariftis; fed vere tamen dicere pofum, fxpius immerito. De fua potius hi querantur imprudentia fibique culpæ dent, quod horreum fit caftum \& promtuarium inane. Coli fidera luftramus, \& a corporibus cceleftibus varias mutationes terræ non immerito colligimus, eorum, quæ juxta nos crefcunt, prorfus negligentes. Arbores referare gemmas foliaque fua explicare quolibet videmus anno; hinc concludimus, experientia fuffulti, ver appropinquare : nemo autem adhuc oftendere potuit, quamnam arborem noftrum calendarium efle voluerit Providentia, ex qua difcamus, quo die femen, in fpem futura mellis, terræ mandare debeat agricola. 


\section{(3) $) 13(103$ \\ b. V.}

Facillimam \& breviffimam viam in operibus fuis lemper fecluitur natura ; qui hane itaque imitari velit, eandem obfervabit methodum. Haut facile quisquam negaverit eandem vim, qux fo. lia arborum producit, fegetes quoque protrudere; fed jure nemo dixerit, pracosem fementem, maturam remper \& ubique acceleraturam effe frugem. Itaque feliciorem nobis haut forre pollicebimur fuccefum, quam fi ex VERNATIONE ARBORUM (e) facionis defumamus fundamentum. Difpiciendum nimirum eft, quo ordine quælibet arbor pro fua fpecie, aëris calore ipfiusque terræ indole folia frua emittat. Collatis deinde plurium annorum oblervationibus non erit difficile ex frondefcentia arborum, fi non pro certo, 1altem maxime probabiliter definire tempus, quo non tantum Hordeum, verum etjam Secale vernssm, Avenam aliasque plantas annuas terræ mandare debeant agricola.

\section{VI.}

Hic ut obtineretur finis, fuere varii , quĭ hortatu Nob. D. Præfidis in variis Patriæ regio. nibus annotaverint non tolum tempus fronde fenthe arborum, fed \& diem, quo Hordeum feve-

rint

(e) Per VERNATIONEM ARBORUM intelligimus fatum illum, quo arbores vi of impul/u nature geminas explicare $\dot{\delta}$ in folia ersinpere acci. piunt. 


\section{(cer) $) \times 4($}

rint \& mefluerint, easque oblervationes benigne nobiscum communicare non gravati fint $(f)$. Sin. gulis Honoracifimorum Virorum ob benevolen. tiam hac in re nobis præltitam, multam debe. mus gratiam, præfertim plurimam D. Torén re. ferimus, qui per triennium in eadem arbore e. jusdem \{peciei, omni cura \& diligentia hafce in. itituit obfervationes; nec non D. Erico Ekelund qui id ipfum pari induftria duobus annis præetitit. Nonnullis autem rempus forfan \& locus (f)

In SCANia circa

Ollandia Par.
Londin.Prof.L.Liedteck pro A.IZ50. Re/mo Paft. P. Weidling - 1750. Calmarie - circa Liungby D. Uddenbergb $1750,175 \mathrm{I}$. In Smonand.Merid. - Notebäck Mag. B. Kallenberg 1750. - Septent. - Gripenberg Ego 1750, 1751,1752. Cir.Gothosurg. ad littus maris. Paft. J. Ableiof $1750,1751$. In Bayusse infula Korn D.S. Lindberg - 1750,1751. NOR VEGLE finit. circ. Nåfinge D.M. Torén $1750,1751,1752$. Wermetand. - Carolit.Lect.J.Fryck/ell 1750,1751. Sudermannie Par. Wingåcker D. L. Willemont 1750. Westman, circ. Aro/.Lect.a.Carlbaum 1750,1751,1752. Uplandia Dalekarlia Finlandia Uplaliam D. E. Ekelund 1750, 75519 Fablunam D. OI. Kalinetter 1750. Aboam Prof. J. Lecbe - 1750 . Ostrobothnile Par. Pybajåcki Paft. N. Matbefius 1750. Medelpad.Par.Stode Com:ft.D.Nordftr8m 1750,1751,1752. Westroboth. circa Pitbea Præp. C. Solander 1750,175L. LAPPONLA Jar. Queckjork Paft.Ol,Modén $1750,1751,275$, 
non femper fuit experimenta fua eadem attentio ne inftituendi; Hilis enim qui urbe detinentur, non raro deeft copia hæc rite obfervandi, \& qui ruri verfantur, domefticis curis ab ejusmodi negotiis fæpe abftrahuntur. Quod fi vero hæ ob. fervationes fecundum has inftitutæ fuiflent regulas, ut, I:mo per citatum triennium, allegatis o. mnibus in locis, inftitutæ fuiflent, idque $2:$ dø circa eadem arborum individua, \& 3:tio circa arbores quæ eadem terra, eodemque gaudene fitu cum agro; tunc forfan certiores regulas coIonis obfervandas formare patiilfemus; cum vero hæ interdum neglectæ fint, non xque bene procedet negotium noftrum; quis en:m ignorat ventum borealem, umbram folumque humidum Vernationem arborum tantum impedire, quantum alcitudo loci, immunitas a feptentrionalis venti furore, \& fitus ad radices montium eam promoveat? Præterea in has obfervationes fxpe irrepfit aliud quoddam vitium. Nempe nonnulla arbores inter quarum Vernationem non plures, quam duo aut tres dies intereffe debent, frpe duarum hebdomadum intervallo a fe disjunguntur, ut reticeam ordinem frondefentix ( $(.3 .3)$, quem vix ac ne vix quidem transgrediuntur arbores, privilegio ipfius naturæ fuffulta, in this diariis ixpe a natura alienum.

\section{VII.}

T mpis, quo arbores frondes furs explicare coperint in varis Pat:ix provinciis, dienque, quo 


\section{(3) $) 16($}

hordeum terræ commiferint meffores Anno MnCCL, fequens montrabit Schema. Defunt quidem obfervata plurimis in locis pro Annis MDCCLI \& MDCCLII, fed fuere, quæ fvalerint, ut non ideo hos duos annos heic omitterem. Divifi Schema illud in duas partes, in quarum urraque prior numerus Romanus menfem,

$$
\begin{aligned}
& \text { ex. gr. II Februarium, IV Aprilem, } \\
& \text { III Martium, V Majum \&x. }
\end{aligned}
$$

inferior Svecus diem indicat, \& præterea fupre: ma, quo die folia fua, unaquaque arbor in mar. gine citata, primum in fingulis Provinciis emiferit, infima autem, tempus, quo non folum feminarint, fed \& mefluerint, \& tandem, quot dies inter fementem \& meffem in unaquaque Provincia interfuerint.

\section{VIII.}

Si annum MDCCL refpicere velimus, folito mitius in his regionibus fæviife hiemem \& ver fuifle valde tempeftivum recordamur. Unde non. nulli in Uplandia turic arva fua confeverunt circiter circa finem menfis Februarii, qui raro alias id prius facere folent, quam inenfe Aprili. Non me quidem fugit, agros quarundam provinciarum bo. realium præmaturam pofcere fementem, præfertim quarum arva conftant terra argillacea, idque vel ideo, ut ipfe ager minori labore fubigatur, \& prima hordei herba terram facilius rumpat, antequam crufta argillacea eam duraverit. Scani autem, alii- 


\section{Anno MDCCL.}

Anno MDCCLI.

Anno MDCCLII.

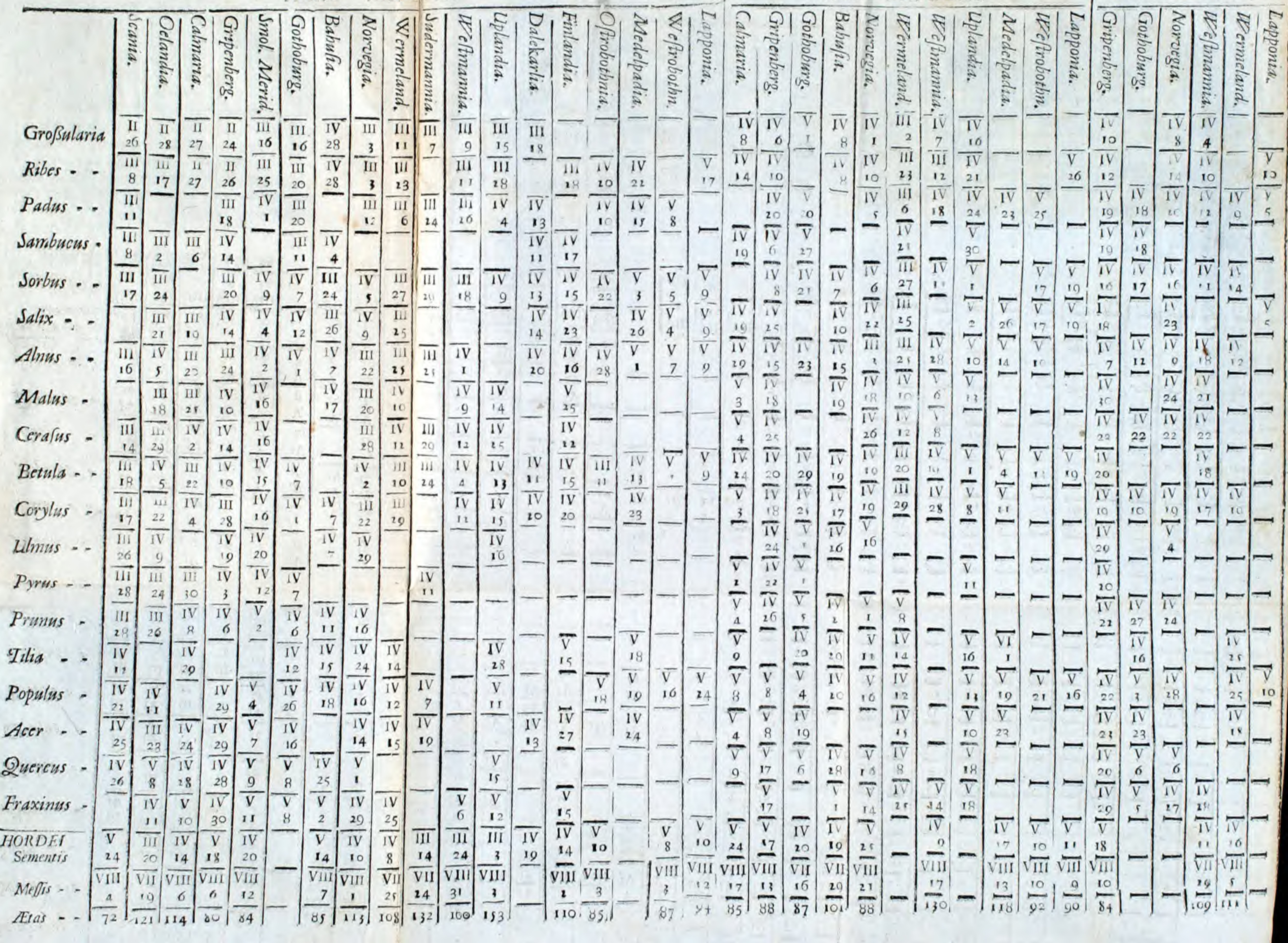





\section{(20) ) I (}

que maris accolæ fero femen agro committunt, five ver fuerit maturum five non, idque interdum cum magno fuo damno, non aliam forte ob cauflam, nifi ut morem fervent, quem a fuis haufere majoribus, Maxime Borealibus Sveciæ Accolis necefum eft femina terræ mandare, quam primum regelata fic terra, fi brevis æf tas perficiat \& abfolvat maturitatem fpicx, antequam bruma incidat. Uti enim ova fixum tempus ad exclufionem pulli requirunt, fic etjam in variis provinciis hordeum ad maturitatem obtinendam; in cujus rei teftimonium adducam aliquot exempla.

A diverfo feminationis tempore concludimus ad tempus æftivum, neceflarium in qualibet provincia ad maturitatem Hordei.

Sementis

Pithox 1740 Maji 24 Meffis Auguf. 21 Dies 89

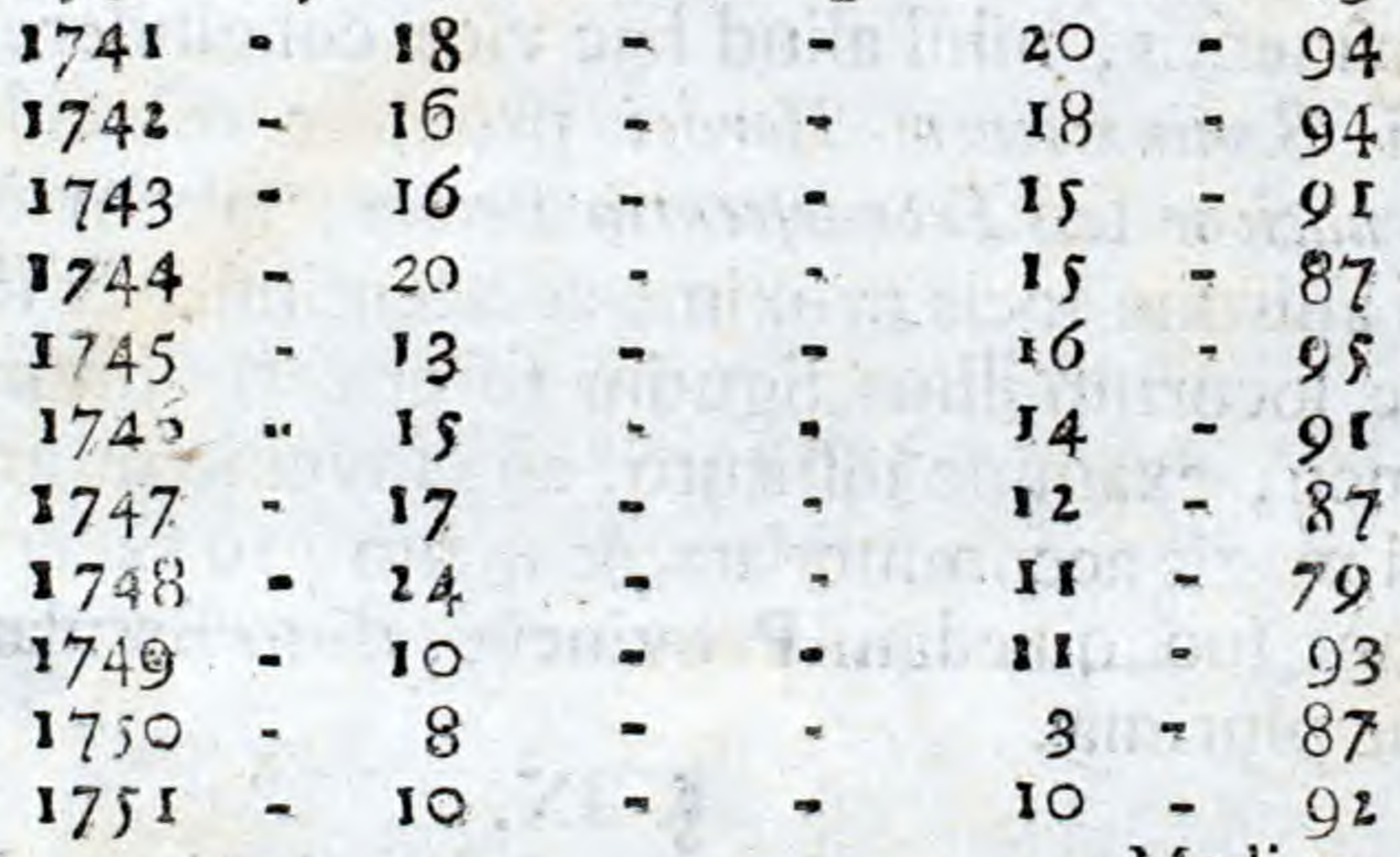

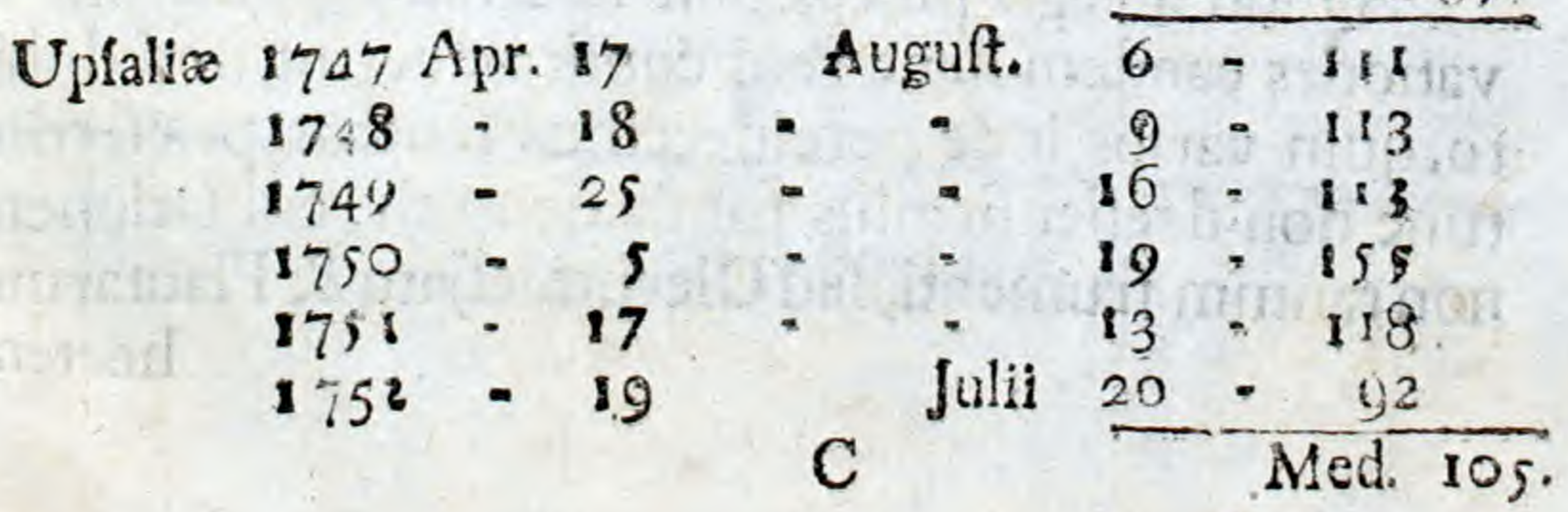




\section{(6) $18($ (e)}

In Nåfinge verfus Norvegiam

1750 Aprilis 9 Auguft. I - II3

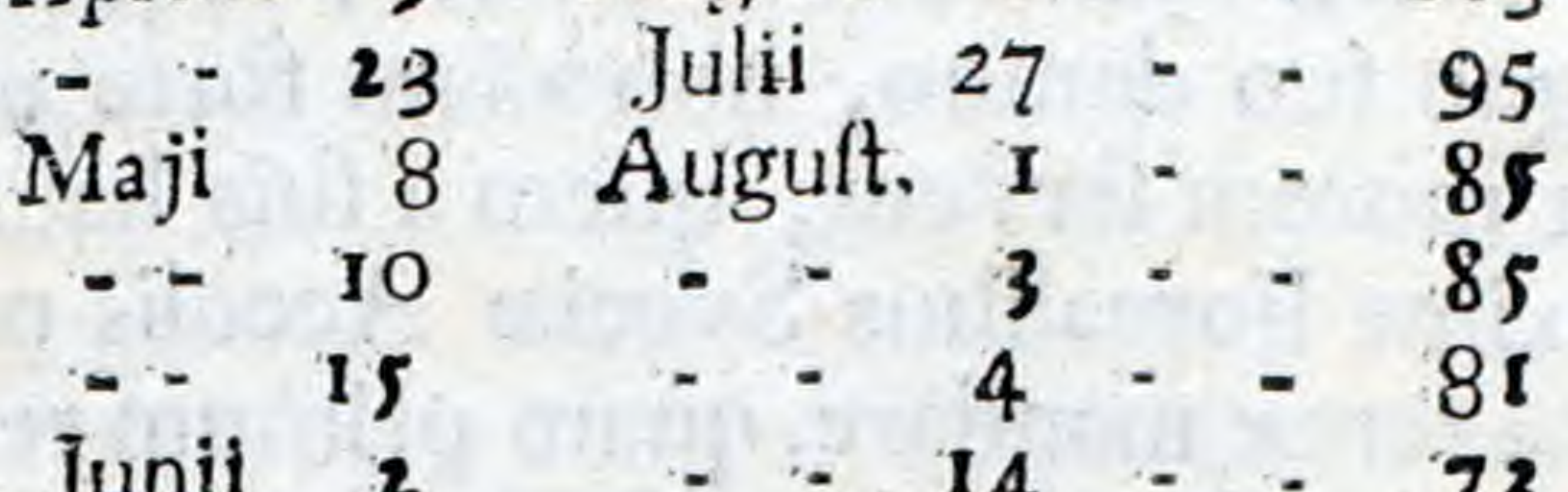

- Kórn Infula Bahufix

I73 Maji 17

1732 Junii 7

Ausuit. 20

Med. 93.

1734 April 28

$1735 \quad$ Maji 14 Septemb. 3 - 88

$1736 \quad-18$

1738

$-23$ Auguit. 7

$$
\begin{aligned}
& \text { - } 4 \text { - } 82 \\
& \text { - } 16-90 \\
& \text { - } 25 \text { - } 94 \\
& \frac{118}{\text { Med. } 100 .}
\end{aligned}
$$

1739 April. 27

Ex his, qux jam attulimus, nec non ex variis aliis ex. perimentis, nihil aliud hac vice concludere poftum, nif Seminationem Hordei proxime coincidere cum Vernatione feu Fronde/centia Betule, faltem in Uplandia aliisque locis proxime adjacentibus; \& fi non ubi. que locorum illius fignum foret certifimum, facile tamen, examine inftituto, alia invenietur arbor huic fini magis accommodata, \& quam pro Calendario habeant fuo quædam Provincix, dum Betulam confulant plurimæ.

\section{IX.}

Quod fi ergo plures poft hac infiruendx obfervationes eandem hane rem confirmaverint, non dubito, quin varios inde perciperemus fructus, præfertim tunc non deeflet firmius fundamentum, cui fationem nón tantum frumenti, fed Olerum etjan \& Plantarum 


\section{(6) 2:9) (13)}

hortenfum attemperaret œeconomus. Quid itaque commodi publico accederet, fi quis in unaquaque Provincia quotannis obfervationes, debita cum attentione, hac in re inftituere, easque demum eodem modo, quo Aftronomi obfervationes fuas Meteorologicas, cum Societate aut Academia Regia Scientiarum communicare vellet? Præterea e re erit obfervafle, quænam fementis, variis diebus veris inftituta, uberio. rem proferat fructum, ut ex hocce collato cum frandefcentia variarum arborum, elucefceret tempus huic negotio maxime adæquatum. Pari modo non inutile foret annotafle, quo in unaquaque Provincia tempore plantæ certæ, faltem prætantiores, florefcant, ut velucior vel tardior anni progreffus inde patefceret; videmus enim, licet hæ in ufu adhuc non fuerint obfervationes, quomodo de tempore FOENICESII, vel ex floribus Parnaffie, Scabiafe fuccife, Gentiane pnetrmonant bes aut Antberici oflifragi prorumpentibus, vel ex floribus Trifolii pratenfis evanefcentibus, feu maturitate feminum Rbinanti, aut in locis elevatis ex fla. vedine foliorum Arnice augurentur fonimeffores.

Profecto fi Botanici, ritu Aftronomorum, annotarent tempus vernationis \& florefcentix arborum atque herbarum, diesque, quibus femen feratur, florefcat \& macurefcat, easque obfervationes plares annos continuarent, nullum effet dubium, quin inde elicere poffemus fundamenta, ex quibus tuto concludere liceret, quo tempore fruges \& plantæ culina. res, pro cuiluslib toli coelique genio, feri deberent. Nec deeflent argumenta, ex quiqus de HIEMIS ad- 


\section{(अ) 20 (}

ventu auguraremur; non ignoraremus ferius ne an ocyus autumnalem faceremus fementem; \& vatem de nique, quem confulerent, tutiffimum haberent hor. tulani,qui lubricam unice jam feqvuntur conjeeturam. ๑. $\mathrm{X}$.

Hæc funt pauca qux in tam vafta materia in medium proferre volui; ne mireris B. L., fi tua ex/pectatione brevior fui in argumento non adeo trito, nec dum ad liquidum perducto. Non mearum fuic virium omnia ad vivum refecare, fed fumma folummo. do rerum faftigia fequendo, anfam folidioribus præbere volui interiores hujus argumenti

\section{receffus ulcerius excutiendi- \\ SOLI DEO GLORIA!}

\section{Domino AUCTORI.}

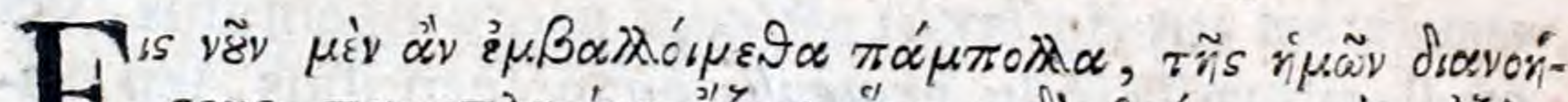

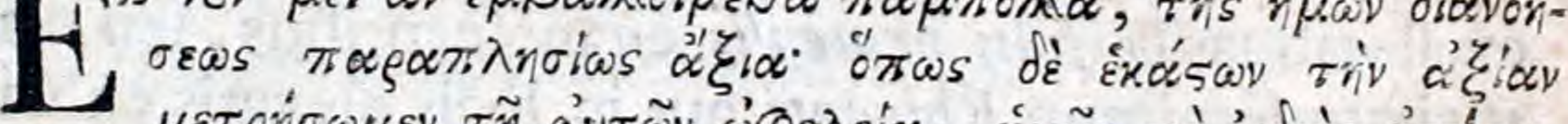

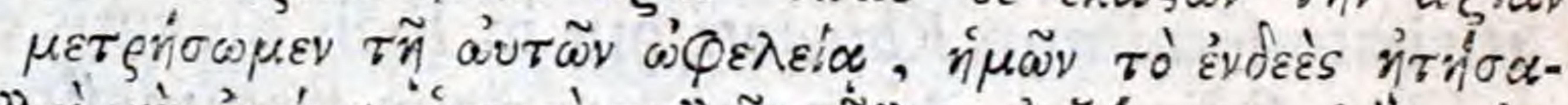

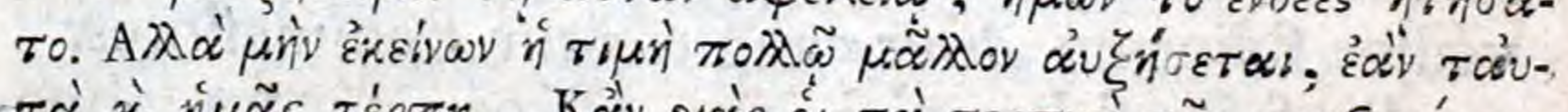

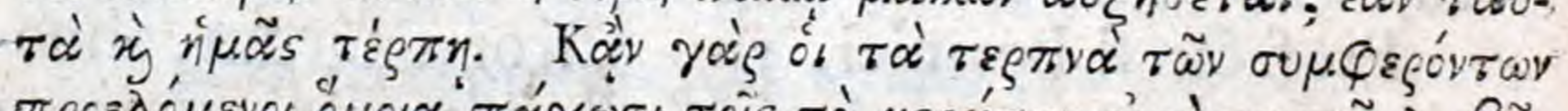

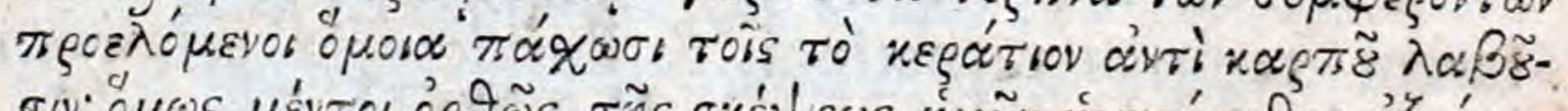

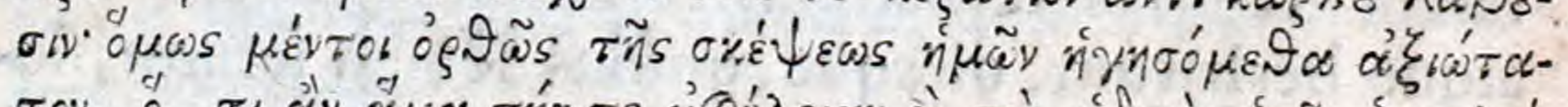

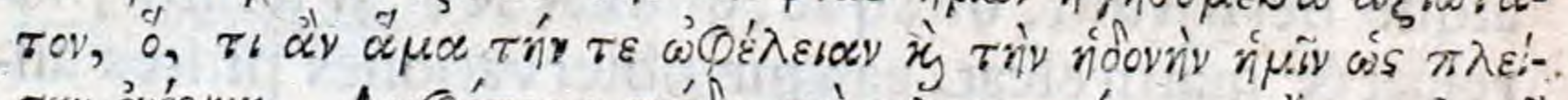

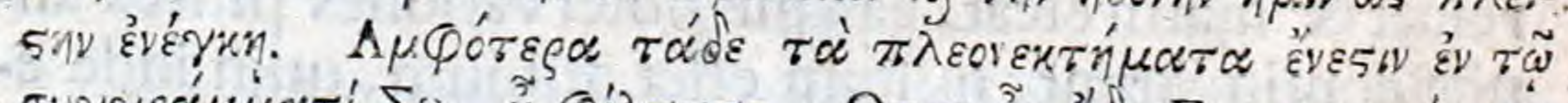

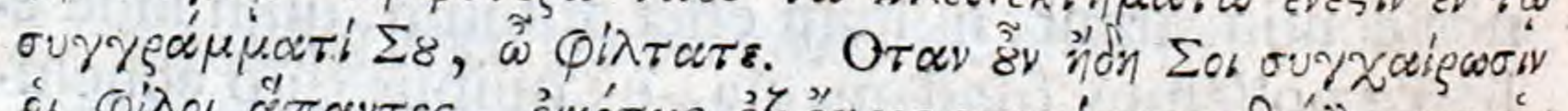

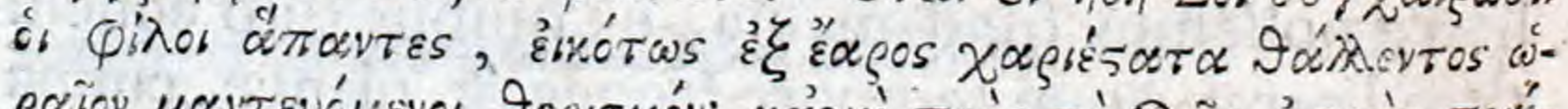

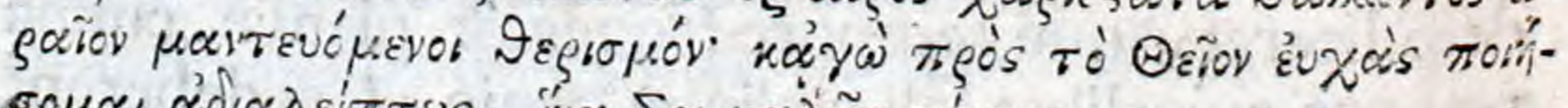

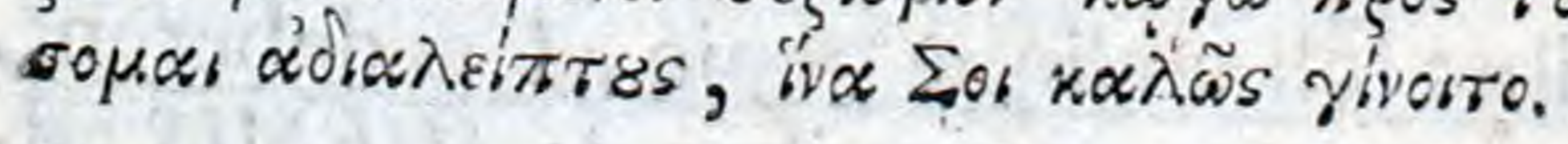

